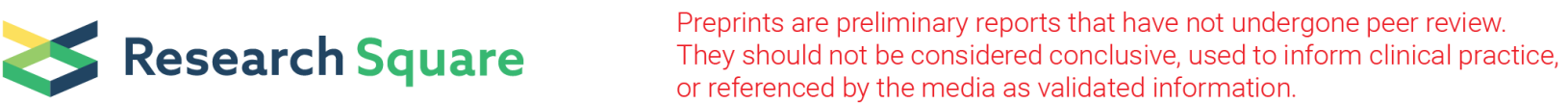

\section{Positive impact of perioperative oral management on the risk of surgical site infections after abdominal surgery: A multicenter retrospective analysis conducted in Japan}

\section{Tetsu Shimane ( $\nabla$ shimanetetsu@gmail.com )}

Shinshu Daigaku - Asahi Campus: Shinshu Daigaku https://orcid.org/0000-0003-1419-9834

Kazuyuki Koike

Chiba Daigaku

Shigeyuki Fujita

Wakayama Kenritsu Ika Daigaku

Hiroshi Kurita

Shinshu Daigaku

Emiko Tanaka Isomura

Osaka Daigaku

Daichi Chikazu

Tokyo Ika Daigaku

Naomi Kanno

Tsukuba Daigaku

Keiichi Sasaki

Tohoku Daigaku

Satoshi Hino

Ehime Daigaku

Hideharu Hibi

Nagoya Daigaku

Takahiro Koyama

Niigata Daigaku

Seiji Nakamura

Kyushu Daigaku

Takeshi Nomura

Tokyo Shika Daigaku

Yoshiyuki Mori

Jichi lka Daigaku

Itaru Tojyo

Wakayama Kenritsu Ika Daigaku 


\section{Toshiro Yamamoto}

Kyoto Furitsu Ika Daigaku

Iku Yamamori

Yamagata Daigaku

Keiko Aota

Tokushima Daigaku

Hideki Tanzawa

Chiba Daigaku

\section{Research Article}

Keywords: surgical site infections, SSI, perioperative oral management, POM, multicenter analysis, general anesthesia

Posted Date: May 4th, 2021

DOI: https://doi.org/10.21203/rs.3.rs-339943/v1

License: (c) (i) This work is licensed under a Creative Commons Attribution 4.0 International License. Read Full License 


\section{Abstract}

Background: Postoperative surgical site infections (SSI) can be major complications, which can prolong postoperative treatment. Perioperative oral management (POM) was first covered by Japanese national health insurance system in 2012, and patients that are scheduled to undergo major surgical treatment receive dental and oral management during the perioperative period.

Objectives: The purpose of the present study was to investigate the ability of POM to prevent perioperative SSI in patients undergoing abdominal surgery.

Methods: The patients' medical records were retrospectively reviewed, and the effects of POM were investigated in a multicenter analysis involving many cases. Detailed information about SSI was assessed and compared between patients with and without POM in univariate and multivariate analyses.

Results: In total, the cases of 2,782 patients (1,750 males 1,032 females) were reviewed. Of these, POM was performed in 778 patients $(28.0 \%)$. Univariate analyses revealed that diabetes mellitus, Eastern Cooperative Oncology Group (ECOG) performance status, the American Society of Anesthesiologists (ASA) classification, the surgical site, the preoperative Prognostic Nutritional Index score, POM, the extent of surgery, the operation time, and the amount of intraoperative blood loss were significantly associated with postoperative SSI (Chi-square or Mann-Whitney U-test, $\mathrm{p}<0.01$ ). The multivariate analysis revealed that POM had significant preventative effects against postoperative SSI (estimate: -0.245 , standard error: $0.080, p<0.01)$. The surgical site, the ASA classification, and the operation time were also significant and independent clinical predictors of SSI.

Conclusion: This study suggests that POM helps to prevent SSI in patients that undergo abdominal surgery.

\section{Introduction}

Surgical site infections (SSI) are associated with increased morbidity and mortality. In addition, SSI have an important influence on the duration of hospitalization and healthcare expenditure, and they also have a negative impact on patients' quality of life [1]. In a report about nosocomial infection surveillance in Japan [2], the incidence of SSI in Japan was estimated to be around 6\%. The latter report revealed that SSI occurred most frequently after abdominal surgery (digestive organ surgery), which accounted for $88 \%$ of all SSI. SSI remain one of the most common infectious complications after abdominal surgery. Thus, measures to prevent SSI are important [3].

The Surgeon General's report on oral health in America showed that oral functional management can prevent general complications during the perioperative period [4]. In Japan, perioperative oral management (POM) was first covered by the national health insurance system in 2012, and patients that are scheduled to undergo surgery for cancer, cardiovascular disease, or organ transplantation receive dental and oral functional management during the perioperative period. POM might help to prevent 
dental-related local and systemic infections in patients that are compromised by invasive surgery. POM has been reported to help prevent postoperative pneumonia, which has been attributed to the aspiration of oral/oropharyngeal pathogens, in some cancer patients ${ }^{4}$. Recently, Nobuhara et al. reported that POM reduced the risk of SSI after colorectal cancer surgery and shortened patients' postoperative hospital stays [5]. The Centers for Disease Control and Prevention (CDC) guidelines recommend that all infections that arise at locations remote from planned surgical sites should be identified and treated before elective operations, and elective operations should be postponed in cases involving patients with remote site infections until the infection has resolved. POM might help to reduce the frequency of SSI after abdominal surgery [6]. However, no large studies into the effects of POM on the risk of SSI have yet been performed.

Therefore, the purpose of the present study was to investigate the ability of POM to prevent SSI in patients who undergo abdominal surgery under general anesthesia, based on a multicenter retrospective analysis of a large number of cases.

\section{Patients And Methods}

The present study was conducted by the Japanese Stomatological Society. The medical records of 2,782 patients who were scheduled to undergo abdominal surgery under general anesthesia between April 2016 and March 2017 at 16 university hospitals were examined in this study. The medical records of these patients were retrospectively reviewed, and data concerning the presence or absence of SSI and clinical factors that might influence the risk of SSI were collected. The presence/absence of SSI was determined based on the documentation in the patients' medical records. The potential predictors of SSI included patient factors (age, sex, smoking habits, diabetes mellitus, severe heart disease, severe pulmonary disease, Eastern Cooperative Oncology Group performance status (ECOG-PS), and the preoperative Prognostic Nutritional Index (PNI)), treatment factors (the risk of general anesthesia, the surgical site, the extent of surgery, the operation time, and the amount of intraoperative blood loss), and the presence/absence of POM. Severe heart disease was defined as $\geq$ grade 3 on the New York Heart Association Functional Classification [7]. Severe pulmonary disease was defined as a percentage vital capacity (VC) of $<60 \%$ or a percentage forced expiratory volume in one second (\%FEV1) of $<50 \%$. The risk of general anesthesia was evaluated with the American Society of Anesthesiologists (ASA) physical status classification [8]. The extent of surgery was divided into 3 groups; i.e., into endoscopic surgery or an incision of $\leq 10 \mathrm{~cm}$, laparotomic surgery or an incision of $>10 \mathrm{~cm}$, or laparostomy and thoracotomy. The surgical sites were divided into 10 groups: the stomach/duodenum, the jejunum/ileum/appendix/colon, the rectum, the liver, the gallbladder/biliary tract, the pancreas, the abdominal wall/abdominal hernias, the peritoneum/retroperitoneum/mesentery/retina, the anus/periphery, and the spleen. The PNI was calculated according to the method reported by Onodera et al [9]. PNI scores were classified into 50-point groups. Operation times were classified into 3-hour groups, and intraoperative blood loss was categorized into 300-ml groups. 
POM was generally initiated from the time when the decision to hospitalize the patient was made. POM included oral health instructions; the removal of dental calculi (scaling); professional mechanical tooth cleaning; the removal of tongue coatings with a toothbrush; the cleaning and adjustment of dentures; and the extraction of teeth affected by severe periodontitis that exhibited pain, pus discharge, mobility, or marked alveolar bone loss on an X-ray examination.

The effects of POM on SSI were investigated statistically via univariate analyses (Chi-square test, MannWhitney U-test) and multivariate analysis (stepwise logistic regression analysis). Statistical analyses were performed using JMP ver.13 (SAS Institute Inc., North Carolina, USA). P-values of $<0.05$ were considered to be significant.

\section{Results}

The patients' characteristics are summarized in Table 1. There were 1,750 males and 1,032 females. Patients aged $40-79$ accounted for $73.4 \%$ of the subjects. As for the ECOG-PS, 1,694 (61.0\%), 535 (19.2\%), 154 (5.5\%), 51 (1.8\%), and 47 (1.7\%) patients exhibited ECOG-PS of 0, 1, 2, 3, and 4, respectively. Regarding the ASA physical status classification, 462 (16.7\%), 1781 (64.0\%), 316 (11.4\%), and 15 (5.3\%) patients were categorized as class 1 , class 2 , class 3 , and class 4 , respectively. A total of 778 patients (28.0\%) underwent POM.

SSI were observed in 275 patients (incidence rate: 9.9\%). The pancreas was the most common site of SSI (incidence: 19.0\%), followed by the gallbladder/biliary tract (14.0\%), and liver (13.2\%) (Table 1).

The results of the univariate analyses are also summarized in Table 1. The presence of SSI was significantly associated with the presence of diabetes mellitus, ECOG-PS, ASA physical status, the surgical site, the preoperative PNI score, the extent of surgery, the operation time, and the amount of intraoperative blood loss. Higher prevalence rates of SSI were associated with diabetes mellitus, higher ECOG-PS scores, higher ASA physical status classes, particular surgical sites, lower preoperative PNI scores, the absence of POM, more invasive surgery, longer operation times, and higher amounts of intraoperative blood loss. Sex, age, severe heart disease, and severe pulmonary disease were not significantly associated with the prevalence of SSI.

The results of the stepwise multivariate analysis are summarized in Table 2. The surgical site, ASA classification, operation time, and use of POM had independent and significant impacts on the prevalence of SSI. The effects of smoking habits on SSI were nearly significant $(p=0.06)$. The obtained results revealed that POM helped to prevent SSI (estimate: -0.245 , standard error: $0.080, p<0.01$, odds ratio: 0.78 ). On the other hand, other variables (surgery involving the pancreas, gallbladder/biliary tract or liver vs. others, odds ratio: 1.30; ASA classification, odds ratio: 1.38/class; and the operation time, odds ratio: 1.60/3 hours) displayed significant negative associations with SSI.

\section{Discussion}


Postoperative SSI are an important marker of surgical quality and are associated with longer hospital stays, higher rates of reoperation and readmission, and increased mortality. Poor survival was observed in patients with various types of cancer who exhibited SSI after surgery. It has also been suggested that SSI are associated with shorter disease-free and overall survival in several types of cancer, such as head and neck cancer, breast cancer, colon cancer, and gastric cancer ${ }^{2}$. Furthermore, Nespoli et al. showed that postoperative infections were associated with a poor 5-year survival rate among colon cancer patients [10]. SSI are one of the most common postoperative morbidities, occurring in $5-40 \%$ of patients who undergo colorectal surgery ${ }^{2}$. In addition, it was reported that SSI occur in $7.6 \%$ of patients after major abdominal surgery. In the present study, SSI arose in $9.9 \%$ of patients, which was compatible with the findings of previous studies. As SSI occur quite frequently, measures aimed at preventing them are required.

Many investigators have studied the factors that influence the prevalence of SSI after abdominal surgery. Togo et al. revealed that SSI were significantly associated with a long operation time, marked intraoperative blood loss, a high blood transfusion requirement, and bile fistulas among liver cancer patients [11]. Mingmei et al. demonstrated that SSI were significantly associated with the type of surgery; the surgical procedure (laparoscopic or open); and hospital size; i.e., the number of beds, in patients with colorectal cancer [12]. Fukuda et al. showed that SSI were significantly associated with blood transfusions, the use of antidiabetic drugs, the use of steroids, the operation time, the ASA classification, the surgical procedure, emergency surgery, and age [13]. In the current study, we retrospectively reviewed the cases of patients who underwent abdominal surgery and tried to identify factors that influence the prevalence of SSI. As a result, we found that hepatic, biliary, or pancreatic surgery; a poorer ASA physical status; and a longer operation time were significantly associated with a higher prevalence of SSI. Most of these risk factors are difficult to control before surgery.

On the other hand, this study revealed that POM interventions had independent, significant, and beneficial impacts on the risk of SSI after abdominal surgery. Recently, Nobuhara et al. reported that POM reduced the risk of SSI after surgery for colorectal cancer ${ }^{4}$. Our findings were compatible with those of the latter study. Other studies have found that preoperative oral care reduced the risk of postoperative pneumonia after surgery for esophageal, lung, or major cancer [14-16]. As the aspiration of oral/oropharyngeal fluid containing pathogenic microorganisms is considered to be the main cause of postoperative pneumonia, it is logical that controlling oral bacteria reduces the risk of postoperative pneumonia. Similarly, our results suggested that the use of POM might significantly reduce the risk of SSI after abdominal surgery.

Gingivitis and periodontal disease provide opportunities for bacterial overgrowth, and the richly vascularized and often ulcerated tissues associated with these diseases are susceptible to bacterial invasion [17]. The bulk of dental plaque, such as biofilm, is composed of microcolonies of oral bacteria [14]. Nobuhara et al. mentioned that the oral cavity is recognized as a significant reservoir of pathogenic microorganisms, which can infect multiple organs [5]. Oral bacteria are known to influence various general diseases, such as pneumonia, cardiovascular disease, cerebrovascular disease, rheumatoid arthritis, preterm or low-weight births, sepsis, carcinogenesis, and non-alcoholic steatohepatitis, and SSI 
$[18,19]$. Therefore, achieving quantitative and qualitative control of oral bacteria via oral healthcare is considered to be important for preventing infectious diseases.

The direct transfer of oral bacteria might cause SSI after head and neck or upper digestive tract surgery, as well as postoperative aspiration pneumonia ${ }^{5}$. In addition, the intravascular invasion of odontogenic bacteria, inflammatory cytokines, and/or endotoxins, and their transport to remote organs through blood vessels or lymph ducts might cause SSI at various surgical sites [5]. It is well known that transient bacteremia often occurs after tooth extraction and tooth brushing. The CDC guidelines suggest that preoperative infectious lesions at remote sites are a risk factor for SSI [6]. In 2012, POM started to be covered by the Japanese national health insurance system, and it has since been widely performed in patients that are scheduled to undergo cancer treatment, organ transplantation, cardiovascular surgery, or orthopedic implant surgery. We assessed the changes in the prevalence of oral bacteria detected in blood cultures before and after the introduction of insurance coverage for POM and found that the introduction of insurance coverage for POM had a beneficial effect on the frequency of systemic infections caused by oral bacteria [20].

In addition, we recently found that POM had significant positive effects on perioperative serum albumin levels in patients that were treated surgically under general anesthesia (submitted for publication). It has been reported that a decreased serum albumin level is an independent risk factor for severe postoperative complications and a poor prognosis [21]. Some researchers have reported that dental infections and poor oral health are closely related to lower serum albumin levels. POM, including oral care, the removal of chronic dental infections, and prosthodontic treatments, has positive effects on serum albumin levels, which might consequently reduce the risk of SSI after abdominal surgery [21].

One of the major advantages of the present study is that it was the first to clarify the beneficial effects of POM on the risk of SSI in patients that undergo abdominal surgery, based on a multicenter retrospective study involving a large number of cases. As for the limitations of the present study, the POM criteria and treatment protocols differed from institute to institute because of the study's retrospective nature. In addition, the POM intervention rate was quite low (28.0\%). However, there are ethical difficulties associated with conducting a prospective randomized control study to evaluate the efficacy of POM because POM has only been covered by the Japanese national health insurance system since 2012, and most Japanese patients now receive POM before undergoing cancer, cardiovascular, or transplant treatment. Furthermore, the protocols for and aims of dental interventions are still subject to debate. The establishment of guidelines for POM in patients that are scheduled to undergo surgery is needed in order to standardize such dental interventions.

\section{Conclusion}

This study suggested that POM has significant beneficial effects on the risk of SSI in patients that undergo abdominal surgery. 


\section{Declarations}

Funding/support

The authors declare no funding aid associated with this manuscript.

Conflict of interest/Disclosure

The authors declare no conflicts of interest associated with this manuscript.

Code availability: The authors declare no code with this manuscript

Authors' contributions

All authors contributed to the study conception and design. Material preparation, data collection and analysis were performed by Kazuyuki Koike, Shigeyuki Fujita, Emiko Tanaka Isomura, Daichi Chikazu, Naomi Kanno, Keiichi Sasaki, Satoshi Hino, Hideharu Hibi, Takahiro Koyama, Seiji Nakamura, Takeshi Nomura, Yoshiyuki Mori, Itaru Tojyo, Toshiro Yamamoto Iku Yamamori, Keiko Aota and Hideki Tanzawa. The first draft of the manuscript was written by Hiroshi Kurita and all authors commented on previous versions of the manuscript. All authors read and approved the final manuscript.

Ethics approval

The study protocol was approved by the committee on medical research of Shinshu University (\#3788).

Consent to participate

Informed consent was obtained from all individual participants included in the study.

Consent for publication

The participant has consented to the submission of the case report to the journal.

Acknowledgments

None

\section{References}

1. Badia JM, Casey AL, Petrosillo N, Hudson P M, Mitchell S A, Crosby C (2017)Impact of surgical site infection on healthcare costs and patient outcomes: a systematic review in six European countries. $J$ Hosp Infect 96: 1-15

2. Ministry of Health, Labour and Welfare; Japan nosocomial infections surveillance (2016) https://www.nihjanis.jp/english/about/index.html. Accessed December 10, 2017 
3. Huh JW, Lee WY, Park YA et al (2019) Oncological outcome of surgical site infection after colorectal cancer surgery. Int. J. Colorectal Dis 34: 277-283

4. Soutome S, Yamamoto S, Funahara M, Hasegawa T, Komori T, Oho T, Umeda M (2016) Preventive effect on postoperative pneumonia of oral health care among patients who undergo esophageal resection: a multi-center retrospective study. Surg Infect (Larchmt) 17: 479-84

5. Nobuhara H, Yanamoto S, Funahara M et al (2018) Effect of perioperative oral management on the prevention of surgical site infection after colorectal cancer surgery. Medicine (Baltimore) 97: e12545

6. Mangram AJ, Horan TC, Pearson ML, et al (1999) Guideline for prevention of surgical site infection, 1999. Centers for Disease Control and Prevention (CDC) hospital infection control practices advisory committee. Am J Infect Control 27:97-132

7. Fisher JD (1972) New York Heart Association Classification. Arch Intern Med. 129:836

8. American Society of Anesthesiologists(ASA) ASA Physical Status Classification System (2014) https://www.asahq.org/standards-and-guidelines/asa-physical-status-classification-system Accessed October 15, 2014

9. Onodera T, Goseki N, Kosaki G (1984) Prognostic nutritional index in gastrointestinal surgery of malnourished cancer patients. Nihon Geka Gakkai Zasshi 85:1001-5.

10. Nespoli A, Gianotti L, Bovo G, Brivio F, Nespoli L, Totis M (2006) Impact of postoperative infections on survival in colon cancer patients. Surg. Infect. (Larchmt). 7 Suppl 2: S41-3

11. Togo S, Matsuo K, Tanaka K, Matsumoto C, Shimizu T, Ueda M, Morioka D, Nagano Y, Endo I, Shimada $\mathrm{H}$ (2007) Perioperative infection control and its effectiveness in hepatectomy patients. $J$ Gastroenterol Hepatol 22: 1942-8

12. Du M, Liu B, Li M, et al (2019) Multicenter surveillance study of surgical site infection and its risk factors in radical resection of colon or rectal carcinoma. BMC Infect Dis 14;19:411

13. Fukuda $H$ (2016) Patient-related risk factors for surgical site infection following eight types of gastrointestinal surgery. J Hosp Infect 93:347-54

14. Ejaz A, Schmidt C, Johnston F, Frank S, Pawlik T (2017) Risk factors and prediction model for inpatient surgical site infection after major abdominal surgery. J. Surg. Res 217: 153-159

15. Akutsu Y, Matsubara H, Shuto K, et al (2010) Pre-operative dental brushing can reduce the risk of postoperative pneumonia in esophageal cancer patients. Surgery 147: 497-502

16. Soutome S, Yanamoto S, Funahara M, et al (2017) Effect of perioperative oral care on prevention of postoperative pneumonia associated with esophageal cancer surgery: A multicenter case-control study with propensity score matching analysis. Medicine (Baltimore) 96:e7436. doi: 10.1097/MD.0000000000007436.

17. Jones DJ, Munro CL, Grap MJ, et al (2010) Oral care and bacteremia risk in mechanically ventilated adults. Heart Lung 39: S57-65

18. Iwata E, Hasegawa T, Yamada SI, et al (2019) Effects of perioperative oral care on prevention of postoperative pneumonia after lung resection: Multicenter retrospective study with propensity score 
matching analysis. Surg 165: 1003-1007

19. Lockhart PB, Brennan MT, Sasser HC, Fox PC, Paster B, Bahrani-Mougeot FK (2008) Bacteremia associated with toothbrushing and dental extraction. Circulation 117: 3118-3125.

20. Miyashita M. Kamijio R, Gibo A et, al (2015) Change in the prevalence of oral bacteria detected in blood cultures before and after the induction of insurance coverage for perioperative oral management. J.J.M.C.P 5;24: 2-8

21. Yamada, S. Koike, K. et.al. Positive effects of perioperative oral management on perioperative serum albumin levels in patients treated surgically under general anesthesia: A multicenter retrospective analysis in Japan. (submitted to $\mathrm{N}$ Engl J Med)

\section{Tables}

Table 1. The patients' characteristics and the results of the univariate analyses.

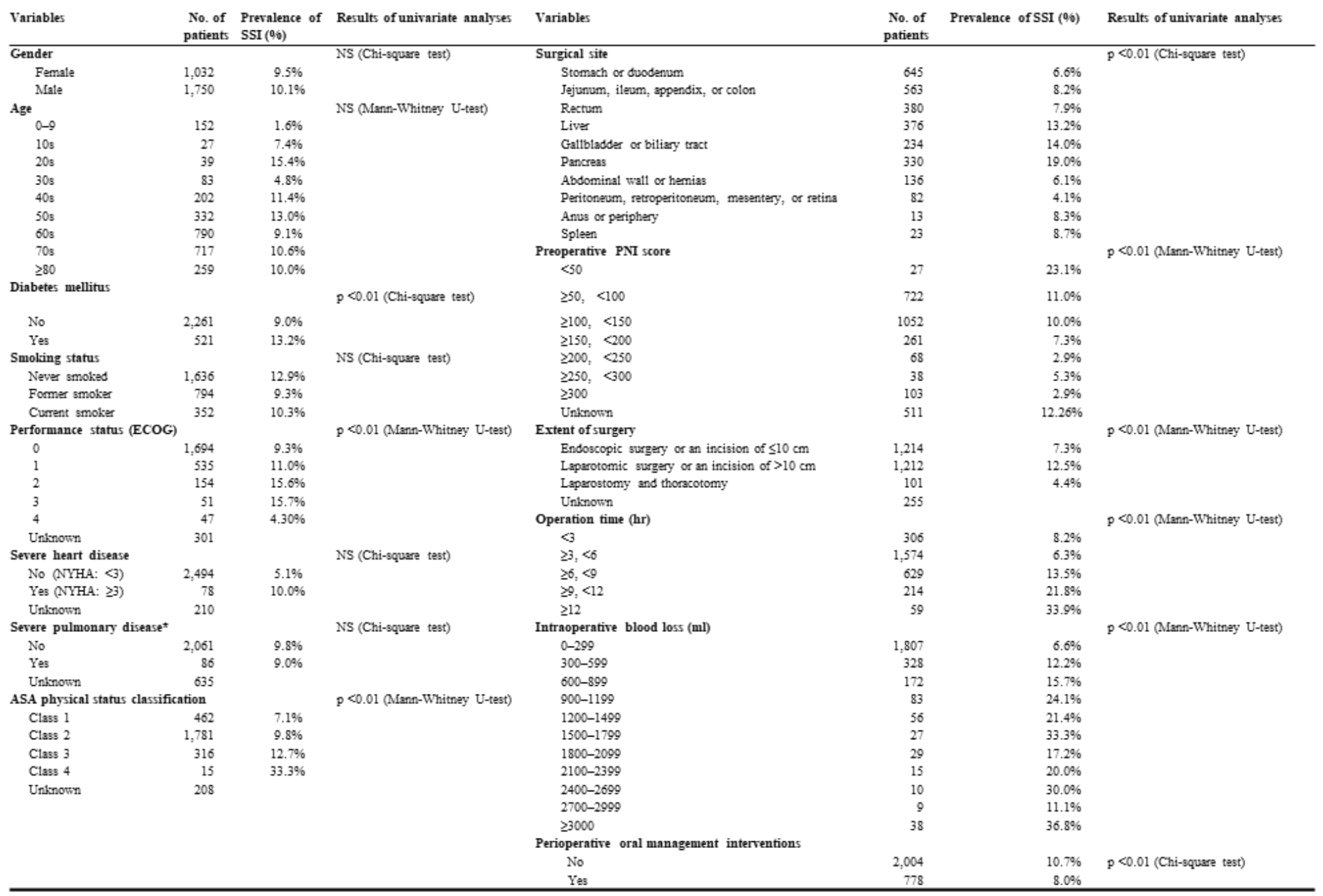

$4:$ severe pulmonary disease

No (\%VC: $\geq 60 \%$ or FEV1.0\%: $\geq 50 \%$ )

Yes (\%VC: $<60 \%$ or FEV1.0\%: <50\%)

Table 2. A multivariate analysis of the effects of POM on the prevalence of surgical site infections among all subjects. 


\begin{tabular}{|c|c|c|c|c|c|c|c|}
\hline & Estimate & Standard error & $\begin{array}{l}\text { Odds } \\
\text { ratio }\end{array}$ & \multicolumn{2}{|c|}{$(95 \% \mathrm{CI})$} & $\begin{array}{c}\text { Chi- } \\
\text { square }\end{array}$ & P-value \\
\hline $\begin{array}{l}\text { Smoking habit (current smoker, former smoker, or never } \\
\text { smoked) }\end{array}$ & 0.234 & 0.123 & 1.26 & 0.98 & 1.59 & 3.63 & 0.06 \\
\hline $\begin{array}{l}\text { Surgical site (pancreas, gallbladder or biliary tract, liver, } \\
\text { or other) }\end{array}$ & 0.265 & 0.073 & 1.30 & 1.13 & 1.50 & 13.32 & $\mathrm{p}<0.01$ \\
\hline ASA classification* & 0.319 & 0.118 & 1.38 & 1.09 & 1.73 & 7.31 & $\mathrm{p}<0.01$ \\
\hline Operation time (every $3 \mathrm{hr}$ ) & 0.473 & 0.070 & 1.60 & 1.40 & 1.84 & 46.11 & $\mathrm{p}<0.01$ \\
\hline POM interventions (yes/no) & -0.245 & 0.080 & 0.78 & 0.67 & 0.91 & 9.44 & $\mathrm{p}<0.01$ \\
\hline
\end{tabular}

*: The American Society of Anesthesiologists (ASA) physical status classification 